Apuntes Universitarios, 2021: 11(4), octubre-diciembre ISSN: 2304-0335 DOI:https://doi.org/10.17162/au.v11i4.760

\title{
Análisis exploratorio de datos espaciales sobre los niveles de logro de aprendizaje en matemática y lectura de los estudiantes de segundo grado de secundaria en la evaluación censal
}

\section{Exploratory Analysis of Spatial Data on the levels of learning achievement in mathematics and reading of second grade secondary students in the Census Assessment}

\author{
Olga Solano Dávila ${ }^{1 a}$, Doris Gómez Ticerán², Grabiela Montes Quintana³, \\ Gregoria Ramón Quispe ${ }^{4}$, Nelly Pillhuaman Caña ${ }^{5}$, \\ Daniel Bolaños Solano ${ }^{6}$
}

Facultad de Ciencias Matemáticas, Universidad Nacional Mayor de San Marcos ${ }^{12345}$ Facultad de Ciencias Empresariales y Educación, Universidad Alas Peruanas ${ }^{6}$

ORCID ID: https://orcid.org/0000-0002-7178-0126 ${ }^{1}$

ORCID ID: https://orcid.org/0000-0001-9253-3661 ${ }^{2}$

ORCID ID: https://orcid.org/0000-0002-9234-7049 3

ORCID ID: https://orcid.org/0000-0001-8996-45594

ORCID ID: https://orcid.org/0000-0002-5767-276X 5

ORCID ID: https://orcid.org/0000-0002-2257-67356

Recibido: 2 febrero de 2021

Aceptado: 18 de mayo de 2021

\begin{abstract}
Resumen
La presente investigación tuvo como objetivo identificar los niveles de logro de aprendizaje en matemática y lectura obtenidos por los estudiantes de segundo grado de secundaria, en la Evaluación Censal de Estudiantes (ECE), durante el año 2018, utilizando el análisis exploratorio de datos espaciales y análisis de componentes principales, y determinar si existe autocorrelación espacial entre los niveles de logro de aprendizaje en matemática y lectura obtenidos por los discentes del segundo grado de secundaria en la ECE en las regiones del Perú. Este estudio se desarrolló dentro de un enfoque cuantitativo el cual se fundamenta en el método hipotético deductivo, no experimental, de diseño transversal porque apuntó en un momento y tiempo definido, el año 2018. El método de recolección de datos que se utilizó fue el de datos secundarios, porque se utilizó la ECE en discentes de segundo grado de secundaria en Perú, el año 2018. Los resultados permitieron determinar el nivel de logro de aprendizaje previo al inicio en matemática y lectura, obtenidos por los estudiantes de segundo grado de secundaria, los más importantes,
\end{abstract}

${ }^{\mathrm{a} C}$ Correspondencia al autor:

E-mail: osolanod@unmsm.edu.pe 
corresponde a las regiones de: Loreto, Amazonas, Ucayali, Huánuco, Huancavelica y Apurímac; con nivel de logro de aprendizaje en inicio en matemática, fueron: Tumbes, Piura, La Libertad, Ica y Callao; con nivel de logro de aprendizaje en inicio en lectura fueron: Tumbes, Piura, Cajamarca, San Martín, Ucayali y Madre de Dios.; asimismo se demostró que existe autocorrelación espacial entre los niveles de logro de aprendizaje en matemática y lectura obtenidos por los discentes del segundo de secundaria en la ECE. $(\mathrm{p}<0.05)$.

Palabras clave: Evaluación de aprendizajes, evaluación censal de estudiantes, estudiantes de segundo grado de secundaria, análisis exploratorio de datos espaciales, análisis de componentes principales, matemática, lectura.

\begin{abstract}
The objective of this research was to identify the levels of learning achievement in mathematics and reading obtained by second grade high school students, in the Student Census Assessment (SCA) in 2018, using the exploratory analysis of spatial data and analysis of main components and to determine if there is spatial autocorrelation between the levels of achievement of learning in mathematics and reading obtained by the students of the second grade of secondary school in the SCA in the regions of Peru. This study was developed within a quantitative approach which is based on the hypothetical deductive, non-experimental, cross-sectional design method because it aimed at a defined time and time, the year 2018. The data collection method that was used was that of secondary data because the SCA was used in second-grade secondary school students in Peru, in 2018. The results allowed determining the level of learning achievement prior to starting in mathematics and reading, obtained by the second grade of secondary school, the most important, corresponding to the regions of: Loreto, Amazonas, Ucayali, Huánuco, Huancavelica and Apurímac; with the level of achievement of learning at the beginning in mathematics, were identified: Tumbes, Piura, La Libertad, Ica y Callao; with the level of learning achievement at the beginning of reading were identified: Tumbes, Piura, Cajamarca, San Martín, Ucayali and Madre de Dios.; It was also shown that there is spatial autocorrelation between the levels of learning achievement in mathematics and reading obtained by second year students in SCA. $(\mathrm{p}<0.05)$.
\end{abstract}

Keywords: Learning assessment, census assessment of students, second grade high school students, exploratory analysis of spatial data, principal component analysis, mathematics, reading.

\title{
Introducción
}

El Ministerio de Educación (MINEDU), todos los años a partir del 2007 realiza la Evaluación Censal de Estudiantes (ECE), para conocer los niveles de logro académico obtenidos por los estudiantes de educación básica. Esta evaluación mide, competencias básicas en comprensión lectora y matemática en todas las instituciones educativas primarias del país. A partir del año 2015, también evalúa a estudiantes de segundo grado de secundaria, en competencias 
básicas sobre comprensión lectora, matemática, historia-geografía y economía y ciencia y tecnología.

De acuerdo al MINEDU (2020), un logro de aprendizaje, previo al inicio, significa que el discente no consiguió los aprendizajes necesarios para estar en el nivel en inicio, sin embargo, un logro de aprendizaje, en inicio, manifiesta que el discente consiguió aprendizajes muy sencillos respecto de lo que se desea para el ciclo evaluado, mientras que un logro de aprendizaje en proceso, representa que el discente consiguió parcialmente los aprendizajes esperados para el ciclo evaluado; se encuentra en camino de lograrlos, pero todavía tiene dificultades, mientras que un logro de aprendizaje satisfactorio, denota que el estudiante logró los aprendizajes necesarios para el ciclo evaluado y está preparado para afrontar los retos del aprendizaje del ciclo siguiente.

En la ECE se evaluaron cuatro campos de aprendizaje, comunicación, matemática, ciencias sociales, y ciencia y tecnología en el año 2018 y en el año 2019 no se consideró al área de ciencias sociales. Los aprendizajes evaluados en estos campos deberían ser desenvueltos por todos los discentes del país al finalizar el sexto ciclo de la educación básica regular (MINEDU, 2018, 2020).

Así, en el campo de comunicación (lectura), lee diferentes tipos de textos escritos en idioma materno. En matemática, soluciona preguntas de cantidad, de regularidad, equivalencia y cambio; problemas de forma, movimiento y localización; problemas de gestión de datos e incertidumbre. En ciencias sociales, construye interpretaciones históricas, gestiona responsablemente el espacio y el ambiente, y gestiona responsablemente los recursos económicos. En ciencia y tecnología, indaga mediante métodos científicos para construir sus conocimientos, explica el mundo físico basándose en conocimientos sobre los seres vivos, materia y energía, biodiversidad, tierra y universo; plantea y edifica soluciones tecnológicas para solucionar problemas de su entorno (MINEDU, 2018, 2020).

Por otro lado, los resultados de la ECE en el año 2019, muestran que algunos logros de aprendizaje en matemática se ha incrementado y en otras ha disminuido comparado con el año 2018, así se tiene que en el nivel de aprendizaje previo al inicio hubo una leve disminución de $0.7 \%$, mientras que en inicio hubo una disminución considerable del $4.3 \%$, en tanto que, en el nivel de logro en proceso, hubo un aumento de solo el 1.4\%; entre tanto que, en el nivel de logro satisfactorio hubo un incremento considerable del $3.6 \%$ de estudiantes que lograron este nivel de logro de aprendizaje (MINEDU, 2020). 
Asimismo, los resultados en lectura en el año 2019 comparado con el año 2018, se tiene que, en el nivel de logro previo al inicio, hubo una disminución leve de $0.8 \%$; en inicio hubo un incremento considerable del $4.5 \%$, en proceso hubo una disminución de $1.9 \%$, y en satisfactorio una disminución de 1.7\%; sin embargo, los resultados en historia-geografía y economía en el año 2018 no se pudo comparar porque en el año 2019, no se consideró esta área (MINEDU, 2020)

En este contexto, el objetivo de la presente investigación fue identificar los niveles de logro de aprendizaje de estudiantes en lectura y matemática obtenidos por los estudiantes de segundo grado de secundaria por regiones del Perú, en la Evaluación Censal de Estudiantes 2018; así como también determinar si existe autocorrelación espacial en los niveles de logro de aprendizaje en matemática y lectura obtenidos por los discentes del segundo de secundaria en la ECE.

\section{Metodología}

\section{Población objetivo}

En las evaluaciones censales 2018, existieron tres poblaciones objetivas: los estudiantes que asistieron a clases en el cuarto de grado de primaria en una IE de Educación Básica Regular (EBR); los que asistieron a clases en el cuarto grado de primaria en una IE que desarrolla el Programa de Educación Intercultural Bilingüe (EIB); y los que asistieron a clases en el segundo grado de secundaria de una IE de Educación Básica Regular (EBR). (MINEDU, 2018). Se tenía programado evaluar a 558674 estudiantes de segundo grado, pero la cobertura fue del $97.40 \%$ (MINEDU, 2018), para el presente artículo se consideró a estudiantes de segundo grado de secundaria matriculados en una IE el año 2018, la muestra que se consideró fue de 538279 estudiantes matriculados en las diversas IE de las regiones del Perú. (MINEDU, 2018).

\section{Instrumentos}

El instrumento de recolección de datos fue una prueba en la que se evaluaron a los estudiantes de segundo grado en las áreas de Matemática y Comunicación. El instrumento midió a través de un puntaje las distintas habilidades y capacidades requeridas para cada área de aprendizaje, posteriormente y tomando como referencia dicho puntaje son clasificados en un nivel de logro. (MINEDU, 2019). A continuación, se presenta el conjunto de competencias a evaluar de acuerdo a cada área: 


\section{Área de matemática:}

- Actúa y piensa matemáticamente en situaciones de cantidad.

- Actúa y piensa matemáticamente en situaciones de regularidad, equivalencia y cambio.

- Actúa y piensa matemáticamente en situaciones de forma, movimiento y localización.

- Actúa y piensa matemáticamente en situaciones de gestión de datos e incertidumbre.

\section{Área de comunicación:}

- Comprensión de textos escritos.

La prueba de matemática cuenta con un total de 90 ítems, los cuales están distribuidos en 2 bloques comunes a todos los cuadernillos y 10 bloques no comunes. De los 90 ítems que tiene la prueba, cada estudiante respondió 50 (MINEDU, 2019). La prueba de lectura de segundo grado de secundaria cuenta con un total de 14 textos de diferente tipo, género, formato y complejidad. A partir de este conjunto de textos, se elaboraron 86 ítems. Dichos textos e ítems se distribuyeron en 2 bloques comunes a todas las formas y 10 bloques no comunes. De los 86 ítems que tiene la prueba, cada estudiante respondió 50 (MINEDU, 2019).

\section{Procedimiento}

El enfoque fue cuantitativo el cual se fundamenta en el método hipotético deductivo, no experimental, de diseño transversal porque apuntó en un momento y tiempo definido, el año 2018. El método de recolección de datos que se utilizó fue: "Datos secundarios (recolectados por otros investigadores)", que según Hernández-Sampieri, y Mendoza (2018), “implica la revisión de documentos, registros públicos y archivos físicos o electrónicos” (p. 291), en la presente investigación se utilizó la Base de Datos de los resultados de la Evaluación Censal Estudiantil (ECE) del Perú (MINEDU, 2018). Se trata de un análisis descriptivo porque se describió a los niveles de logro de aprendizaje en matemática y lectura obtenidos por los estudiantes de segundo grado de secundaria por regiones del Perú, en la Evaluación Censal de Estudiantes 2018.

\section{Análisis exploratorio de datos espaciales}

El análisis exploratorio de datos espaciales es un conjunto de técnicas para describir y visualizar la distribución espacial, descubrir patrones de asociación espacial, identificar las observaciones atípicas espaciales y sugerir otras formas de heterogeneidad espacial. (Anselin, 
1996). Los métodos gráficos del AEDE según la tendencia espacial son los siguientes: mapa temático, mapa dinámico, gráficos condicionales, diagrama de dispersión de Morán y diagrama de dispersión de Morán multivariante. Mientras que, entre los métodos gráficos según atípicos espaciales se encuentra el mapa de caja, mapa de percentiles, cartograma, gráficos de LISA y gráficos LISA multivariantes (Chasco, 2003).

\section{Análisis exploratorio de datos espaciales sobre los niveles de logro de aprendizaje en matemática y lectura}

Para identificar los niveles de logro de aprendizaje en matemática y lectura obtenidos por los discentes de segundo de secundaria por regiones del Perú en la ECE, se utilizó el mapa de cuantiles, los datos se dividen y agrupan en una serie de categorías (cuantiles) con igual número de datos, el número de categorías es cuatro y toma el nombre de cuartiles, en este caso el programa Geoda considera el nivel de logro de aprendizaje en matemática o lectura obtenido por los discentes por regiones (Tablas 1 y 2) (De Corso, Pinilla \& Gallego, 2017; Oyana \& Margai, 2015; Buzai \& Baxendale, 2009; Celemín, 2009; Cervera, Lizárraga \& Sánchez 2008; Chasco, 2003).

\section{Tabla 1}

Regiones según nivel de logro de aprendizaje en matemática en estudiantes de segundo de secundaria en el Perú - 2018

\begin{tabular}{lccccc}
\hline \multirow{2}{*}{ Región } & \multicolumn{2}{c}{ Nivel de logro de aprendizaje en matemática } & \multicolumn{2}{c}{$\%$} \\
\cline { 2 - 4 } & Previo al inicio & En Inicio & En proceso & Satisfactorio & Evaluados \\
\hline Amazonas & 50.10 & 30.30 & 11.60 & 8.01 & 100.00 \\
Ancash & 41.80 & 34.70 & 13.30 & 10.20 & 100.00 \\
Apurímac & 53.70 & 30.40 & 9.39 & 6.50 & 100.00 \\
Arequipa & 16.80 & 35.60 & 22.00 & 25.60 & 100.00 \\
Ayacucho & 42.60 & 35.40 & 12.80 & 9.20 & 100.00 \\
Cajamarca & 43.20 & 36.30 & 11.80 & 8.70 & 100.00 \\
Callao & 23.60 & 40.40 & 19.30 & 16.70 & 100.00 \\
Cusco & 39.70 & 34.90 & 14.10 & 11.30 & 100.00 \\
Huancavelica & 51.80 & 32.70 & 9.50 & 6.00 & 100.00 \\
Huánuco & 49.90 & 34.40 & 9.50 & 6.20 & 100.00 \\
Ica & 27.40 & 38.90 & 17.30 & 16.40 & 100.00 \\
Junín & 26.10 & 35.80 & 18.50 & 19.60 & 100.00 \\
La libertad & 33.00 & 38.70 & 16.00 & 12.30 & 100.00 \\
Lambayeque & 34.30 & 38.50 & 14.70 & 12.50 & 100.00 \\
Lima & 21.52 & 38.60 & 20.35 & 19.54 & 100.00 \\
Loreto & 71.40 & 22.80 & 3.90 & 1.90 & 100.00 \\
Madre de Dios & 45.91 & 35.70 & 11.61 & 6.78 & 100.00 \\
Moquegua & 13.41 & 32.79 & 22.40 & 31.40 & 100.00
\end{tabular}




\begin{tabular}{lccccc} 
Pasco & 35.59 & 36.40 & 15.31 & 12.69 & 100.00 \\
Piura & 33.10 & 40.00 & 15.50 & 11.40 & 100.00 \\
Puno & 41.30 & 34.90 & 13.20 & 10.60 & 100.00 \\
San Martín & 49.50 & 34.50 & 9.90 & 6.11 & 100.00 \\
Tacna & 13.80 & 33.10 & 22.11 & 31.00 & 100.00 \\
Tumbes & 43.61 & 39.40 & 10.91 & 6.09 & 100.00 \\
Ucayali & 58.10 & 29.90 & 8.00 & 4.00 & 100.00 \\
\hline
\end{tabular}

\section{Tabla 2}

Regiones según nivel de logro de aprendizaje en lectura en estudiantes de segundo de secundaria en el Perú - 2018

\begin{tabular}{|c|c|c|c|c|c|}
\hline \multirow{2}{*}{ Región } & \multicolumn{4}{|c|}{ Nivel de logro de aprendizaje en lectura } & \multirow{2}{*}{$\begin{array}{c}\% \\
\text { Evaluados }\end{array}$} \\
\hline & Previo al inicio & En Inicio & En proceso & Satisfactorio & \\
\hline Amazonas & 37.10 & 34.50 & 20.10 & 8.30 & 100.00 \\
\hline Ancash & 25.50 & 38.70 & 24.00 & 11.80 & 100.00 \\
\hline Apurímac & 37.30 & 38.40 & 16.90 & 7.41 & 100.00 \\
\hline Arequipa & 6.90 & 30.30 & 35.30 & 27.50 & 100.00 \\
\hline Ayacucho & 27.10 & 40.90 & 21.80 & 10.20 & 100.00 \\
\hline Cajamarca & 28.80 & 42.00 & 20.80 & 8.60 & 100.00 \\
\hline Callao & 7.90 & 34.90 & 36.50 & 20.70 & 100.00 \\
\hline Cusco & 25.20 & 38.40 & 23.90 & 12.50 & 100.00 \\
\hline Huancavelica & 38.40 & 41.20 & 14.89 & 5.50 & 100.00 \\
\hline Huánuco & 32.60 & 40.90 & 19.20 & 7.30 & 100.00 \\
\hline Ica & 12.40 & 38.70 & 31.40 & 17.50 & 100.00 \\
\hline Junín & 15.50 & 37.80 & 29.40 & 17.30 & 100.00 \\
\hline La libertad & 17.70 & 39.30 & 28.10 & 14.90 & 100.00 \\
\hline Lambayeque & 17.40 & 40.30 & 27.80 & 14.50 & 100.00 \\
\hline Lima & 8.30 & 33.63 & 34.13 & 23.95 & 100.00 \\
\hline Loreto & 44.80 & 37.70 & 13.40 & 4.10 & 100.00 \\
\hline Madre de Dios & 22.30 & 44.69 & 24.89 & 8.13 & 100.00 \\
\hline Moquegua & 5.58 & 27.99 & 36.24 & 30.19 & 100.00 \\
\hline Pasco & 21.41 & 40.70 & 26.20 & 11.70 & 100.00 \\
\hline Piura & 16.60 & 42.20 & 27.50 & 13.70 & 100.00 \\
\hline Puno & 27.90 & 40.80 & 21.20 & 10.11 & 100.00 \\
\hline San Martín & 25.90 & 45.00 & 21.20 & 7.89 & 100.00 \\
\hline Tacna & 5.80 & 26.89 & 36.20 & 31.10 & 100.00 \\
\hline Tumbes & 22.00 & 44.49 & 24.19 & 9.32 & 100.00 \\
\hline Ucayali & 31.80 & 42.30 & 19.30 & 6.60 & 100.00 \\
\hline
\end{tabular}




\section{Análisis de componentes principales sobre los niveles de logro de aprendizaje en matemática y lectura}

Se aplicó el análisis de componentes principales para la clasificación del logro de aprendizaje por niveles de logro de aprendizaje en matemática y por regiones del Perú (Tablas 3 y 4, Figuras 13 y 14), asimismo, se aplicó el análisis de componentes principales para la clasificación del logro de aprendizaje por niveles de aprendizaje en lectura y por regiones del Perú (Tablas 5 y 6, Figuras 15 y 16). (Pérez, 2014; Johnson \& Wichern, 2007; Uriel \& Aldás, 2005; Levy \& Varela, 2003).

\section{Tabla 3}

Matriz de componentes principales del nivel de aprendizaje en matemática en estudiantes de segundo de secundaria en el Perú - 2018

\begin{tabular}{lcc}
\hline Nivel de logro de & \multicolumn{2}{c}{ Componente } \\
\cline { 2 - 3 } aprendizaje & 1 & 2 \\
\hline Previo al inicio & -0.999 & 0.032 \\
En Inicio & 0.622 & 0.782 \\
En proceso & 0.991 & -0.100 \\
Satisfactorio & 0.920 & -0.387 \\
\hline Método de extracción: análisis de componentes principales.
\end{tabular}

\section{Tabla 4}

Regiones según puntuaciones factoriales del Análisis de Componentes Principales, del nivel de aprendizaje en matemática en estudiantes de segundo de secundaria en el Perú - 2018

\begin{tabular}{lcc}
\hline \multicolumn{1}{c}{ Región } & \multicolumn{2}{c}{ Puntuaciones factoriales } \\
\hline Amazonas & 1 & 2 \\
Ancash & -0.81 & -0.84 \\
Apurímac & -0.22 & 0.10 \\
Arequipa & -1.08 & -0.65 \\
Ayacucho & 1.48 & -0.96 \\
Cajamarca & -0.27 & 0.36 \\
Callao & -0.32 & 0.65 \\
Cusco & 1.08 & 0.95 \\
Huancavelica & -0.07 & 0.05 \\
Huánuco & -0.93 & -0.02 \\
Ica & -0.80 & 0.40 \\
Junín & 0.78 & 0.64 \\
La libertad & 0.85 & -0.40 \\
Lambayeque & 0.42 & 0.90 \\
Lima & 0.30 & 0.88 \\
& 1.20 & 0.26
\end{tabular}




\begin{tabular}{lcc} 
Loreto & -2.36 & -2.12 \\
Madre de Dios & -0.49 & 0.63 \\
Moquegua & 1.65 & -2.08 \\
Pasco & 0.22 & 0.31 \\
Piura & 0.41 & 1.31 \\
Puno & -0.19 & 0.12 \\
San Martín & -0.77 & 0.42 \\
Tacna & 1.63 & -1.96 \\
Tumbes & -0.33 & 1.65 \\
Ucayali & -1.38 & -0.56 \\
\hline
\end{tabular}

\section{Índice de Moran}

Para el análisis espacial fue utilizado el programa GeoDa versión 1.8 (GeoDa Centro de Análisis Geoespacial y Computación de la Universidad Estatal de Arizona, Tempe, AZ, EUA), con las regiones del Perú según nivel de logro de aprendizaje, en matemática y lectura, obtenido por los discentes en la ECE (Ver Tablas 1 y 2), el índice global de Moran fue usado para explorar la concentración espacial del país con un nivel de significancia de 0.05 , que probó la hipótesis de autocorrelación espacial de los niveles de logro de aprendizaje en matemática y lectura obtenidos por los discentes de segundo grado de secundaria por regiones del Perú, en la Evaluación Censal de Estudiantes 2018.

Los resultados del índice varían entre -1 y +1 , categorizados en: a) valores positivos, de autocorrelación espacial mayores que cero, b) distribución aleatoria, para valores iguales a cero y c) dispersión espacial, para valores debajo de cero (Gordziejczuk \& Lucero 2019; De Corso, Pinilla \& Gallego, 2017; Chue, 2016; García, 2011; Rodríguez, et al., 2010; Griffith, 2009; Acevedo \& Velásquez 2008; Vilalta, 2005; Moreno \& Vayá, 2002; Anselin, 1995; Getis \& Ord, 1992).

\section{Análisis estadístico}

A los resultados de la ECE de los estudiantes de segundo grado de secundaria se les aplicó el análisis exploratorio de datos espaciales, con la finalidad de analizar los niveles de logro de aprendizaje obtenidos por los estudiantes de segundo de secundaria en matemática, y lectura; y luego determinar si existe autocorrelación espacial en los datos. Asimismo, se utilizó el análisis de componentes principales a los niveles de logro de aprendizaje de matemática y lectura obtenidos por los estudiantes de segundo de secundaria por regiones del Perú. 


\section{Resultados}

Con el producto obtenido de la ECE, las regiones según niveles de logro de aprendizaje previo al inicio y en inicio en matemática obtenidos por los estudiantes de segundo de secundaria en el Perú, 2018 (ver Tabla 1), fueron graficadas el mapa de los cuantiles y se muestra en la figura 1. Se observa que, en el mapa de cuantiles para los discentes que lograron un nivel de aprendizaje previo al inicio en matemática, la posición que corresponde al rango más alto, pertenecen a las regiones: Loreto, Amazonas, Ucayali, Huánuco, Huancavelica y Apurímac, estas regiones con mayor porcentaje de estudiantes son coloreados en marrón más intenso (ver figura 1, izquierda; Tabla 1); resultados similares se encontraron con el análisis de componentes principales (ver figuras 13 y 14; Tablas 3 y 4).

Asimismo, se observa que, el mapa de cuantiles para los discentes que lograron un nivel de aprendizaje en inicio en matemática, la posición que corresponde al rango más alto, pertenece a las regiones: Tumbes, Piura, La Libertad, Lima e Ica, estas regiones con mayor porcentaje de estudiantes son coloreados en marrón más intenso (ver figura 1, derecha). Sin embargo, en la Tabla 1 se observa que además de las regiones mencionadas sobresale también la región del Callao, el cual no se muestra en el gráfico porque se encuentra ubicado dentro de la región Lima; por otro lado, los resultados que se encontraron en el análisis de componentes principales coinciden solo con las regiones de Tumbes, Piura y La Libertad, y se suman las regiones de Lambayeque y el Callao, sin embargo no se consideraron las regiones de Lima e Ica en los resultados con este análisis. (ver figuras 5 y 6; Tablas 3 y 4). 


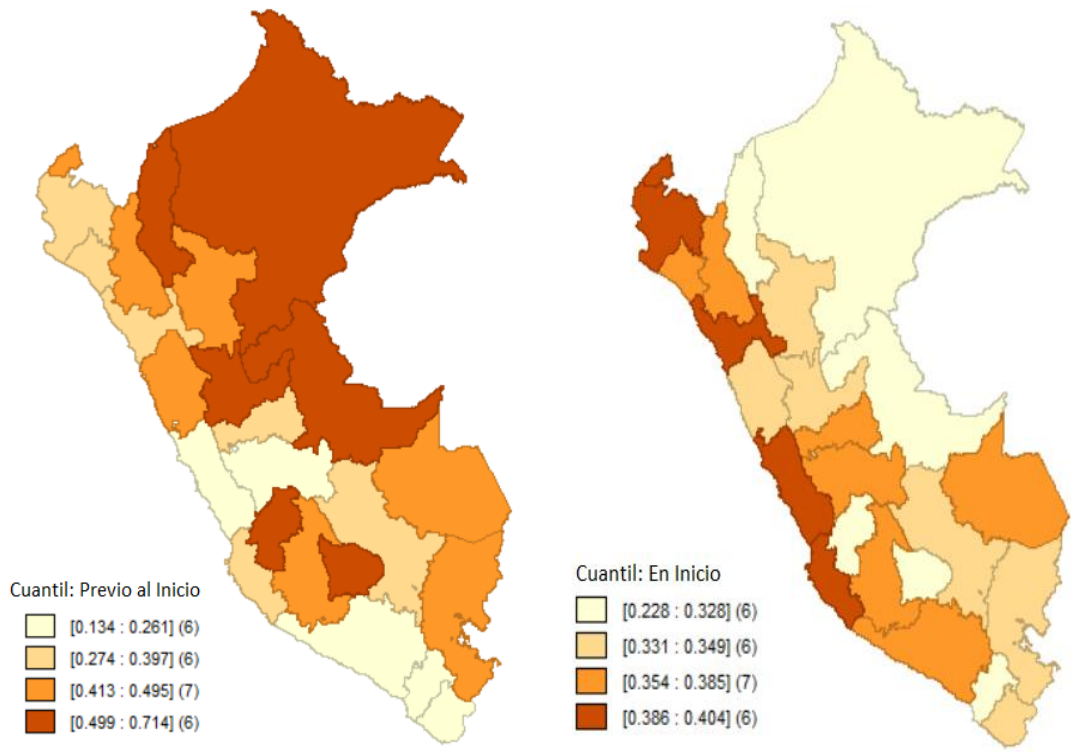

Figura 1. Mapa de cuantiles de los niveles de logro de aprendizaje previo al inicio (izquierda) y en inicio (derecha) en matemática obtenido por los estudiantes de segundo de secundaria en el Perú - 2018.

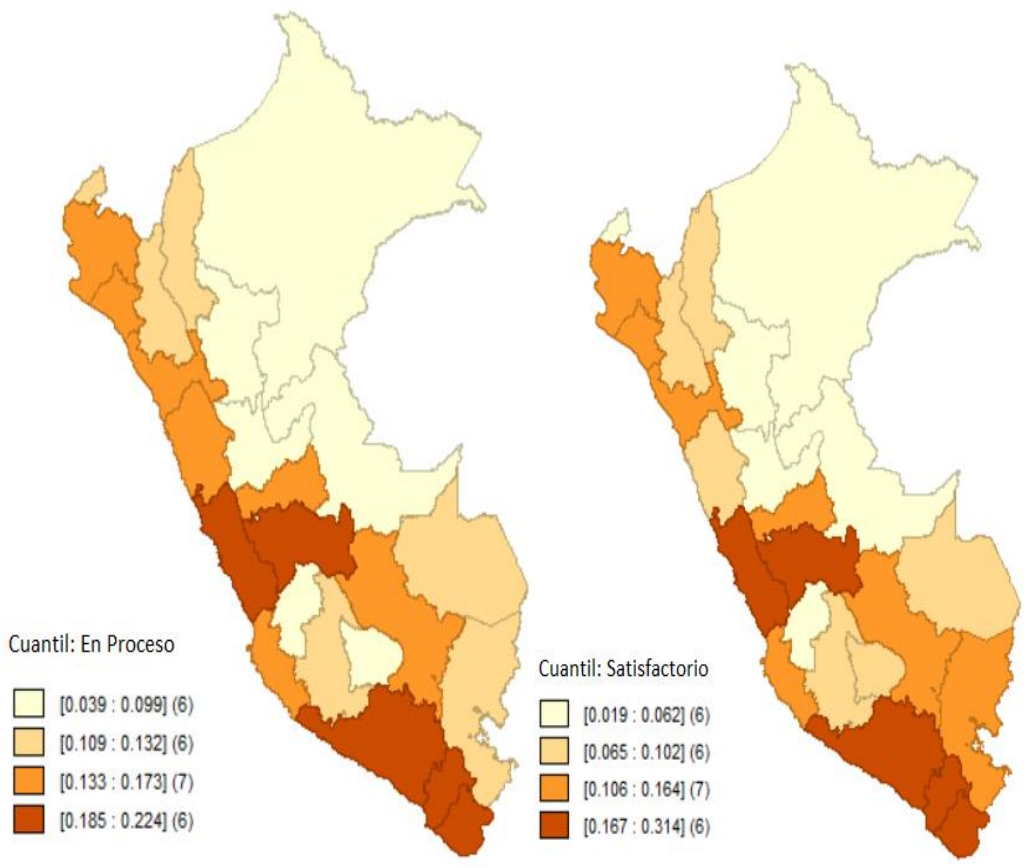

Figura 2. Mapa de cuantiles de los niveles de logro de aprendizaje en proceso (izquierda) y satisfactorio (derecha) en matemática obtenido por los estudiantes de segundo de secundaria en el Perú - 2018. 
Con el producto de la ECE, las regiones del Perú según niveles de logro de aprendizaje en proceso y satisfactorio en matemática obtenidos por los estudiantes de segundo de secundaria en el Perú, 2018, fueron graficadas en el mapa de los cuantiles y se muestran en la figura 2. En el mapa de cuantiles para los discentes que lograron niveles de aprendizaje en proceso y satisfactorio en matemática, la posición que corresponde al rango más alto pertenece a las regiones: Lima, Junín, Arequipa, Moquegua y Tacna, estas regiones con mayor porcentaje son coloreados en marrón más intenso. (ver figura 2). Sin embargo, en la Tabla 1 se observa que además de las regiones mencionadas sobresale también la región del Callao, el cual no se muestra en el gráfico porque se encuentra ubicado dentro de la región Lima; resultados similares se encontraron con el análisis de componentes principales donde se suma la región del Callao. (ver figuras 13 y 14; Tablas 3 y 4 ).

El índice global de autocorrelación espacial de Moran, para los cuatro niveles de aprendizaje en matemática resultaron ser mayores a cero, 0.32, 0.34, 0.31, 0.36, respectivamente, con $\mathrm{p}<0.05$, lo cual demostró que existe autocorrelación espacial con los cuatro niveles de aprendizaje en matemática obtenidos por los dicentes del segundo grado de secundaria en la ECE en las regiones del Perú. (ver figuras 3,4,5, y 6).
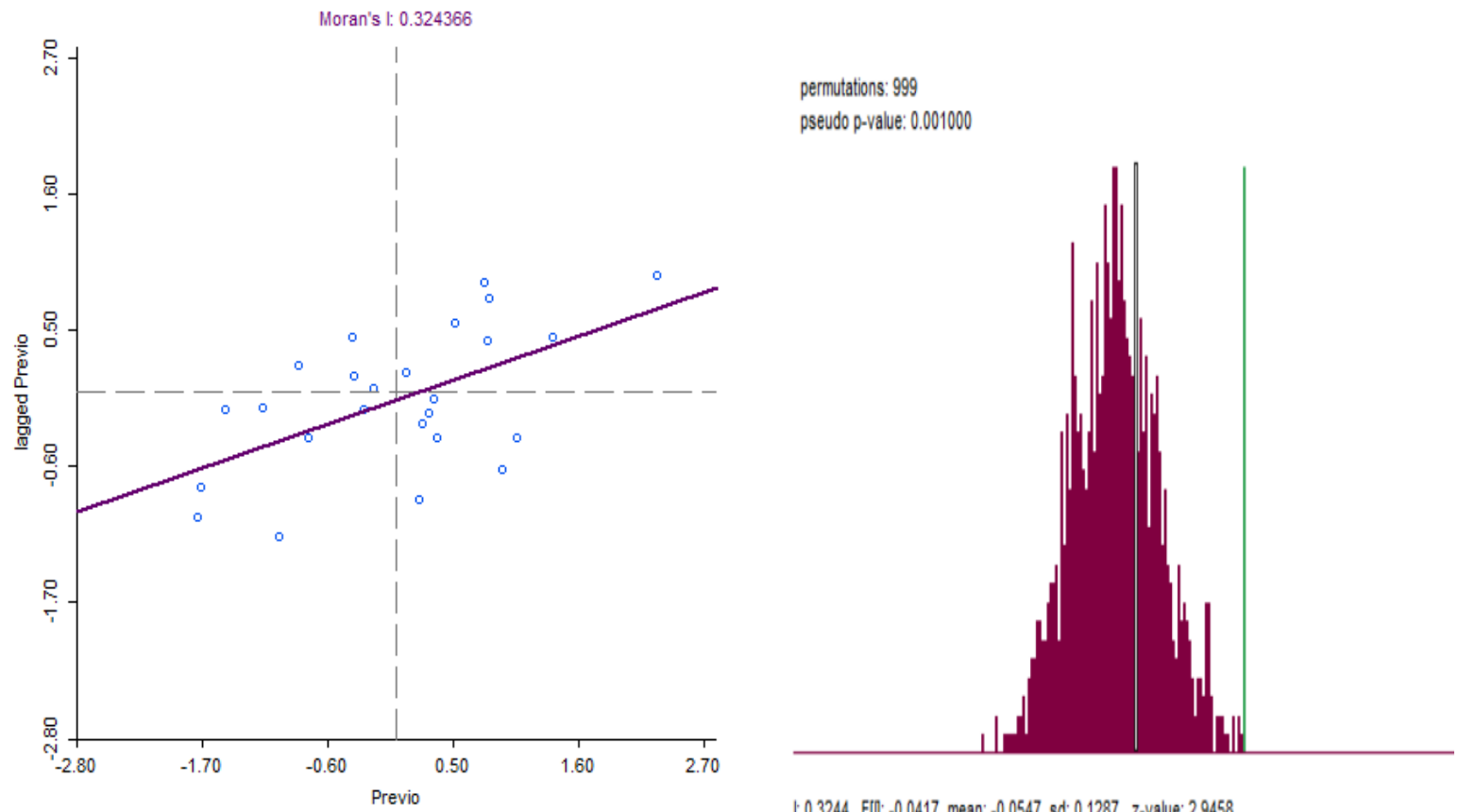

1:0.3244 E[]:-0.0417 mean: -0.0547 sd: 0.1287 z-value: 2.9458

Figura 3. Diagrama de dispersión del Índice de Moran del nivel de logro de aprendizaje previo al inicio en matemática obtenido por los estudiantes de segundo de secundaria en el Perú - 2018. 

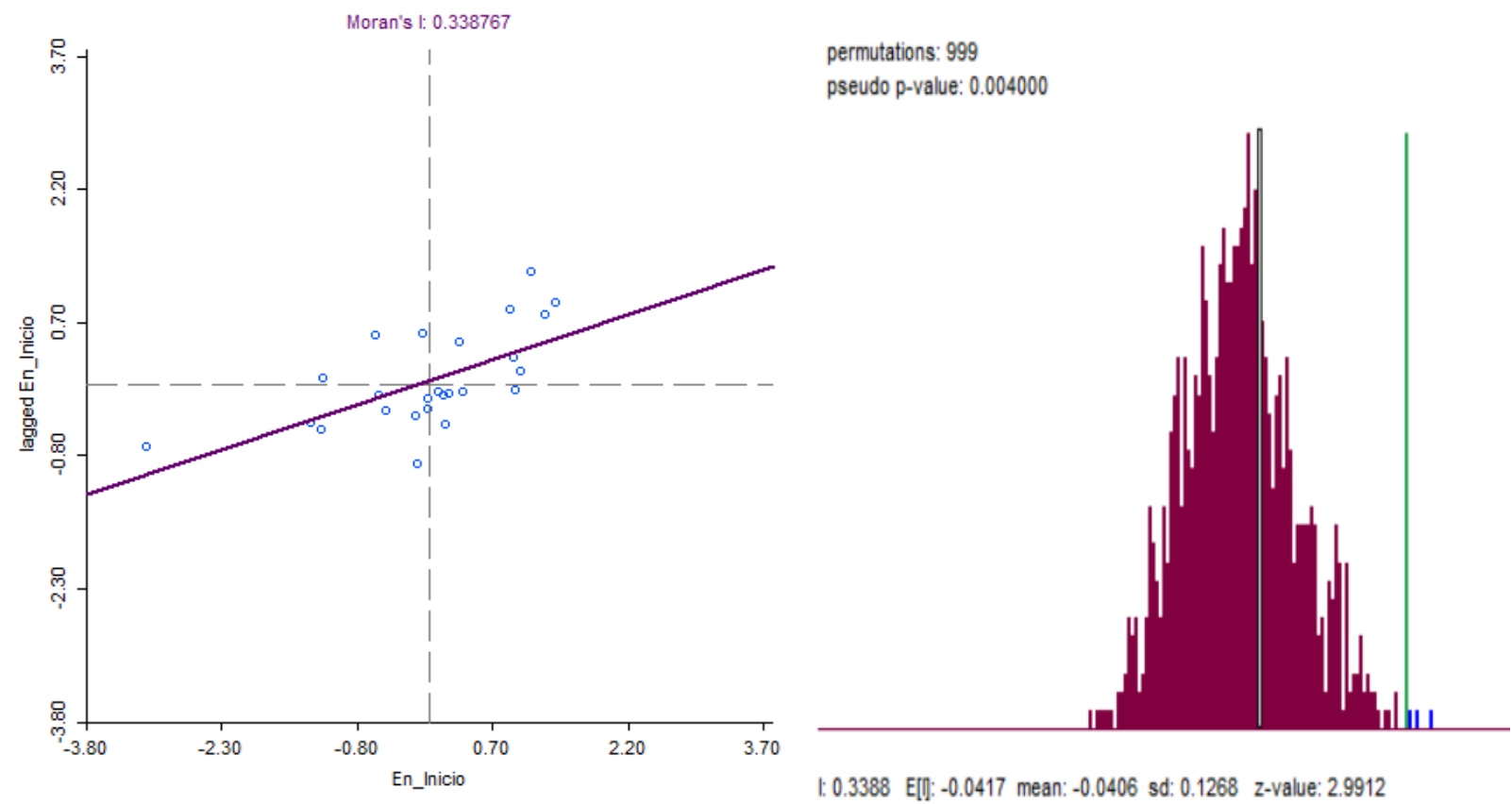

1: 0.3388 E[]; -0.0417 mean: -0.0406 sd: 0.1268 z-value: 2.9912

Figura 4. Diagrama de dispersión del Índice de Moran del nivel de logro de aprendizaje en inicio en matemática obtenido por los estudiantes de segundo de secundaria en el Perú - 2018.
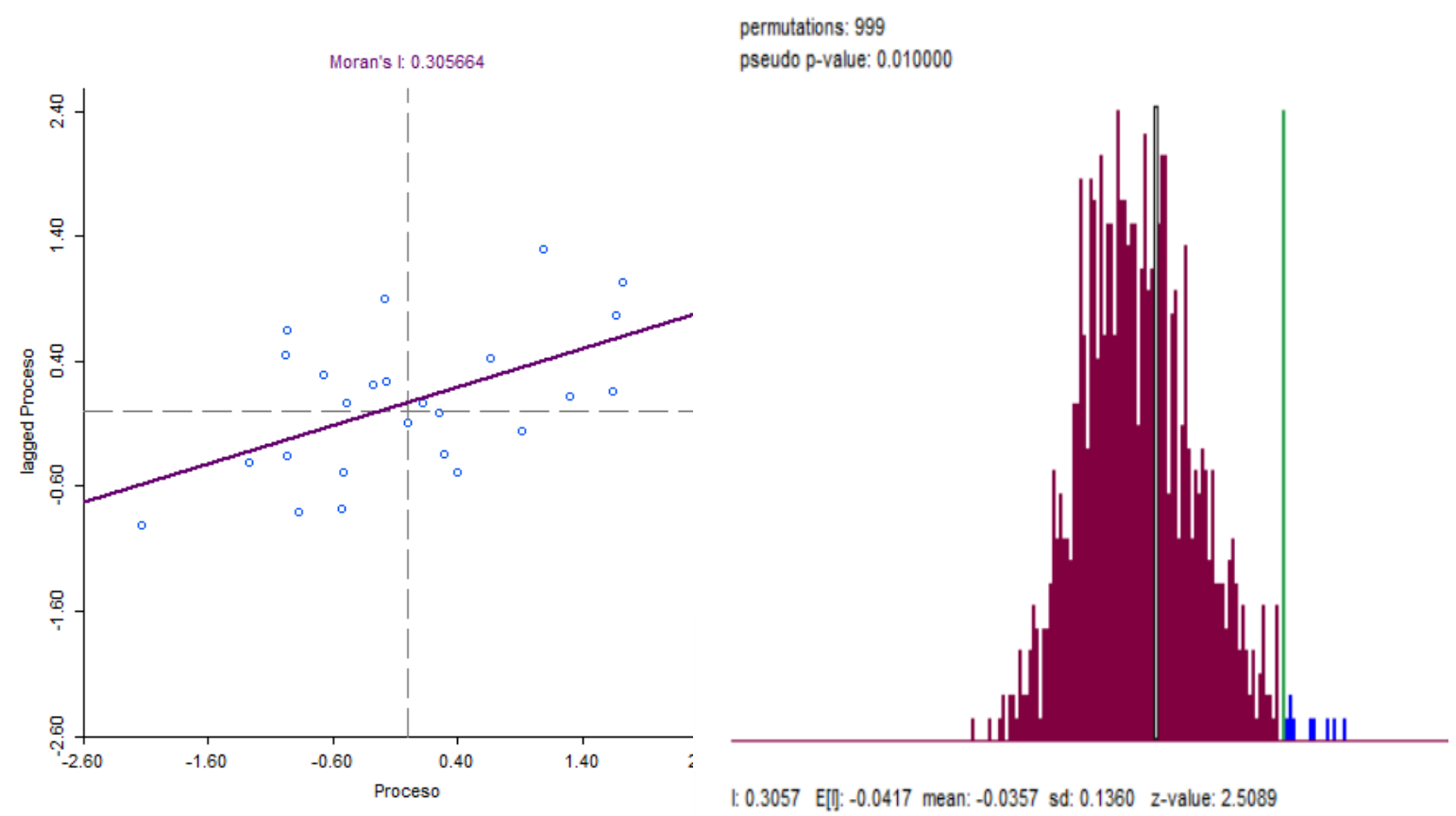

Figura 5. Diagrama de dispersión del Índice de Moran del nivel de logro de aprendizaje en proceso en matemática obtenido por los estudiantes de segundo de secundaria en el Perú - 2018. 


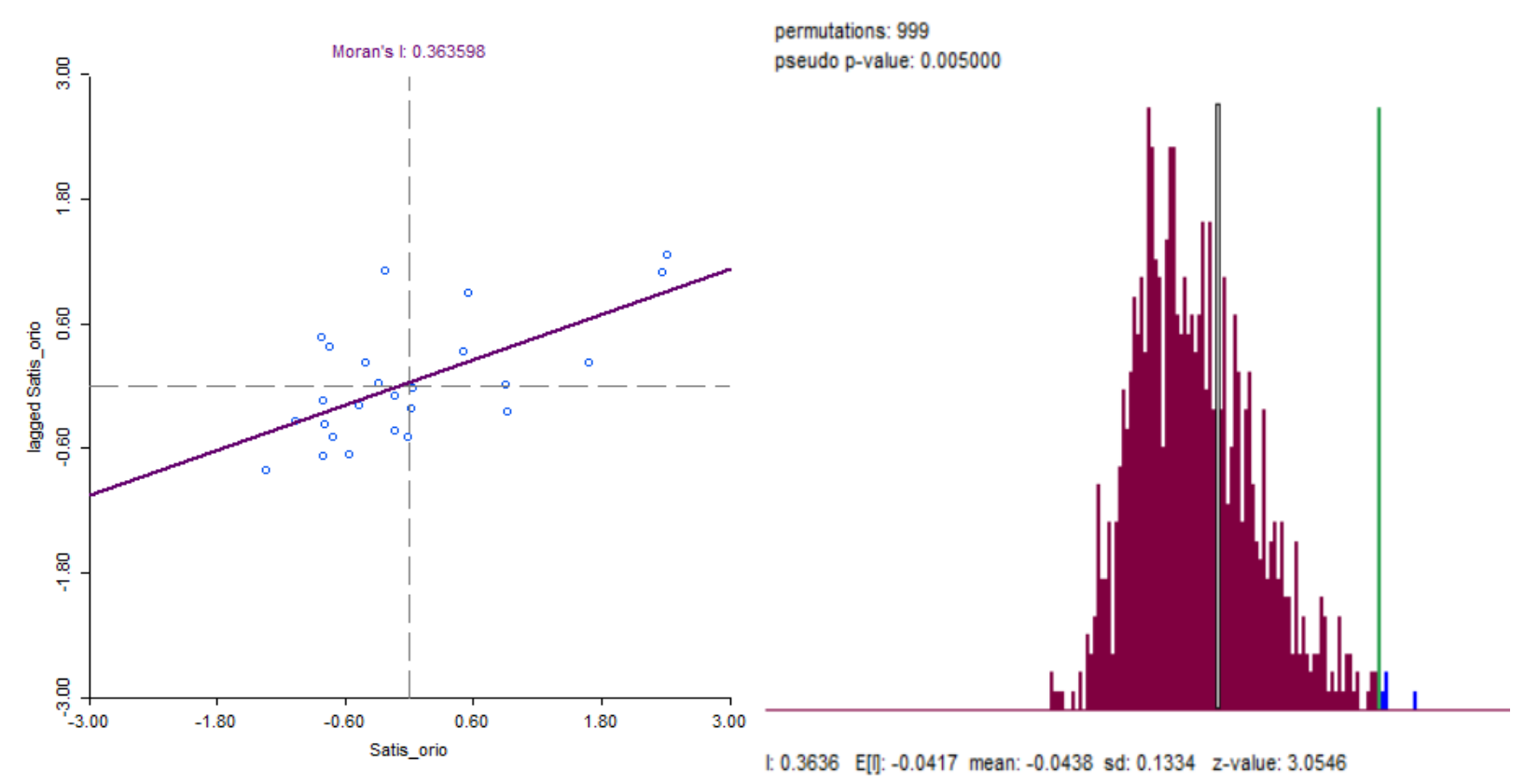

Figura 6. Diagrama de dispersión del Índice de Moran del nivel de logro de aprendizaje satisfactorio en matemática obtenido por los estudiantes de segundo de secundaria en el Perú - 2018.

En la figura 7 muestran el mapa de los cuantiles de los niveles de logro de aprendizaje previo al inicio y en inicio en lectura considerando las regiones del Perú.

Se observa que, en el mapa de cuantiles para el nivel de aprendizaje previo al inicio en lectura, la posición que corresponde al rango más alto, corresponde a las regiones: Loreto, Amazonas, Ucayali, Huánuco, Huancavelica y Apurímac, estas regiones con superior porcentaje de observaciones son pintados en marrón más intenso (ver figura 7, lado izquierdo; Tabla 2); resultados similares se encontraron con el análisis de componentes principales, excepto por la región de Ucayali. (ver figuras 15 y 16; Tablas 5 y 6).

\section{Tabla 5}

Matriz de componentes principales rotados del nivel de aprendizaje en lectura en estudiantes de segundo de secundaria en el Perú - 2018

\begin{tabular}{lcc}
\hline Nivel de logro & \multicolumn{2}{c}{ Componente } \\
\cline { 2 - 3 } de aprendizaje & 1 & 2 \\
\hline Previo al inicio & -0.966 & 0.256 \\
En Inicio & -0.293 & 0.955 \\
En proceso & 0.922 & -0.374 \\
Satisfactorio & 0.763 & -0.638 \\
\hline
\end{tabular}


Método de extracción: análisis de componentes principales.

Método de rotación: Varimax con normalización Kaiser.

\section{Tabla 6}

Regiones según puntuaciones factoriales del Análisis de Componentes Principales, considerando el nivel de aprendizaje en lectura en estudiantes de segundo de secundaria en el Perú- 2018

\begin{tabular}{lcc}
\hline \multirow{2}{*}{\multicolumn{1}{c}{ Región }} & \multicolumn{2}{c}{ Puntuaciones factoriales } \\
\cline { 2 - 3 } Amazonas & -1.64 & 2 \\
Ancash & -0.28 & -1.28 \\
Apurímac & -1.52 & -0.02 \\
Arequipa & 1.05 & -0.50 \\
Ayacucho & -0.34 & -1.49 \\
Cajamarca & -0.45 & 0.39 \\
Callao & 1.37 & 0.56 \\
Cusco & -0.28 & -0.26 \\
Huancavelica & -1.50 & -0.11 \\
Huánuco & -0.88 & 0.08 \\
Ica & 1.06 & 0.25 \\
Junín & 0.67 & 0.39 \\
La libertad & 0.55 & 0.05 \\
Lambayeque & 0.63 & 0.35 \\
Lima & 1.13 & 0.58 \\
Loreto & -2.30 & -0.72 \\
Madre de Dios & 0.44 & -0.87 \\
Moquegua & 1.02 & 1.52 \\
Pasco & 0.28 & -2.01 \\
Piura & 0.81 & 0.60 \\
Puno & -0.44 & 1.04 \\
San Martín & 0.02 & 0.33 \\
Tacna & 0.92 & 1.38 \\
Tumbes & 0.42 & -2.29 \\
Ucayali & -0.71 & 1.42 \\
\hline
\end{tabular}

Asimismo, se observa que, en el mapa de cuantiles para el nivel de aprendizaje en inicio en lectura, la posición que corresponde al rango más alto corresponde a las regiones: Tumbes, Piura, Cajamarca, San Martín, Ucayali y Madre de Dios, estas regiones con porcentaje superior de observaciones son pintados en marrón más intensa (ver figura 7, lado derecho; Tabla 2); resultados 
similares se encontraron con el análisis de componentes principales, excepto por las regiones de Cajamarca y Ucayali (ver figuras 15 y 16; Tablas 5 y 6).
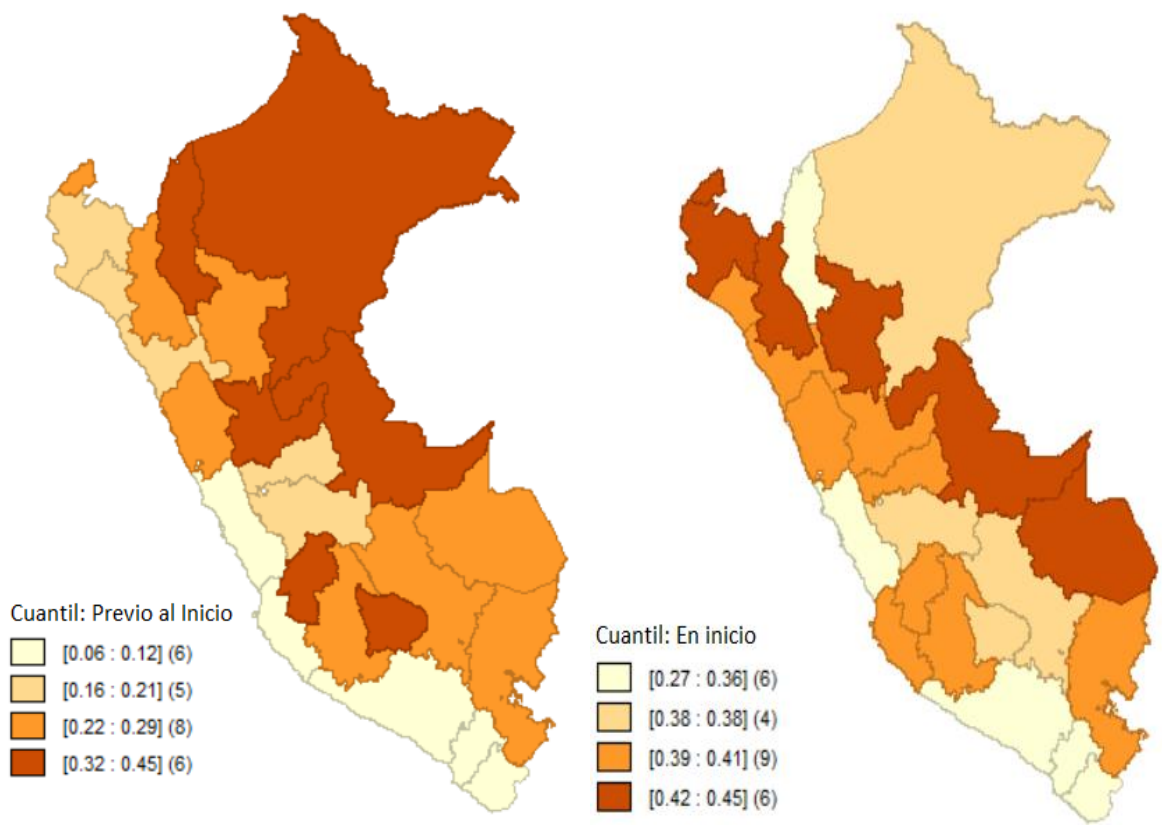

Figura 7. Mapa de cuantiles de los niveles de logro de aprendizaje previo al inicio (izquierda) y en inicio (derecha) en lectura obtenido por los estudiantes de segundo de secundaria en el Perú - 2018
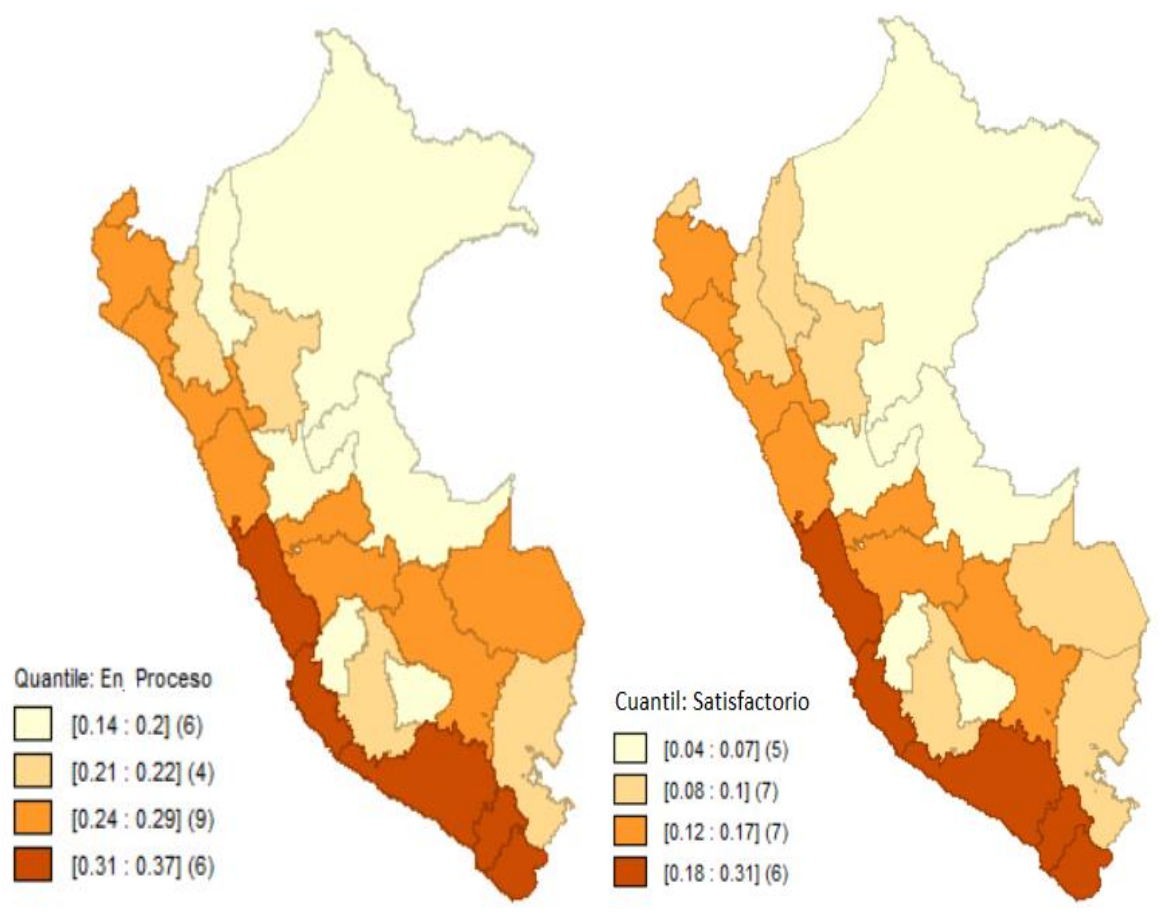

Figura 8. Mapa de cuantiles de los niveles de logro de aprendizaje en proceso (derecha) y satisfactorio (izquierda) en lectura obtenido por los estudiantes de segundo de secundaria en el Perú - 2018 
En la figura 8 se muestran el mapa de cuantiles de los niveles de logro de aprendizaje en proceso y satisfactorio en lectura obtenidos por los estudiantes en la ECE, considerando las regiones del Perú. Se observa que, en el mapa de cuantiles que los discentes que lograron un nivel de aprendizaje en proceso y satisfactorio en lectura, la posición que corresponde al rango más alto pertenece a las regiones: Lima, Ica, Arequipa, Moquegua y Tacna, estas regiones con mayor rango son coloreados en marrón más intenso (ver figura 8). Sin embargo, en la Tabla 2 se observa que además de las regiones mencionadas sobresale también la región del Callao, el cual no se muestra en el gráfico porque se encuentra ubicado dentro de la región Lima; resultados similares se encontraron con el análisis de componentes principales, a estos resultados se suma la región de Callao. (ver figuras 15 y 16; Tablas 5 y 6).
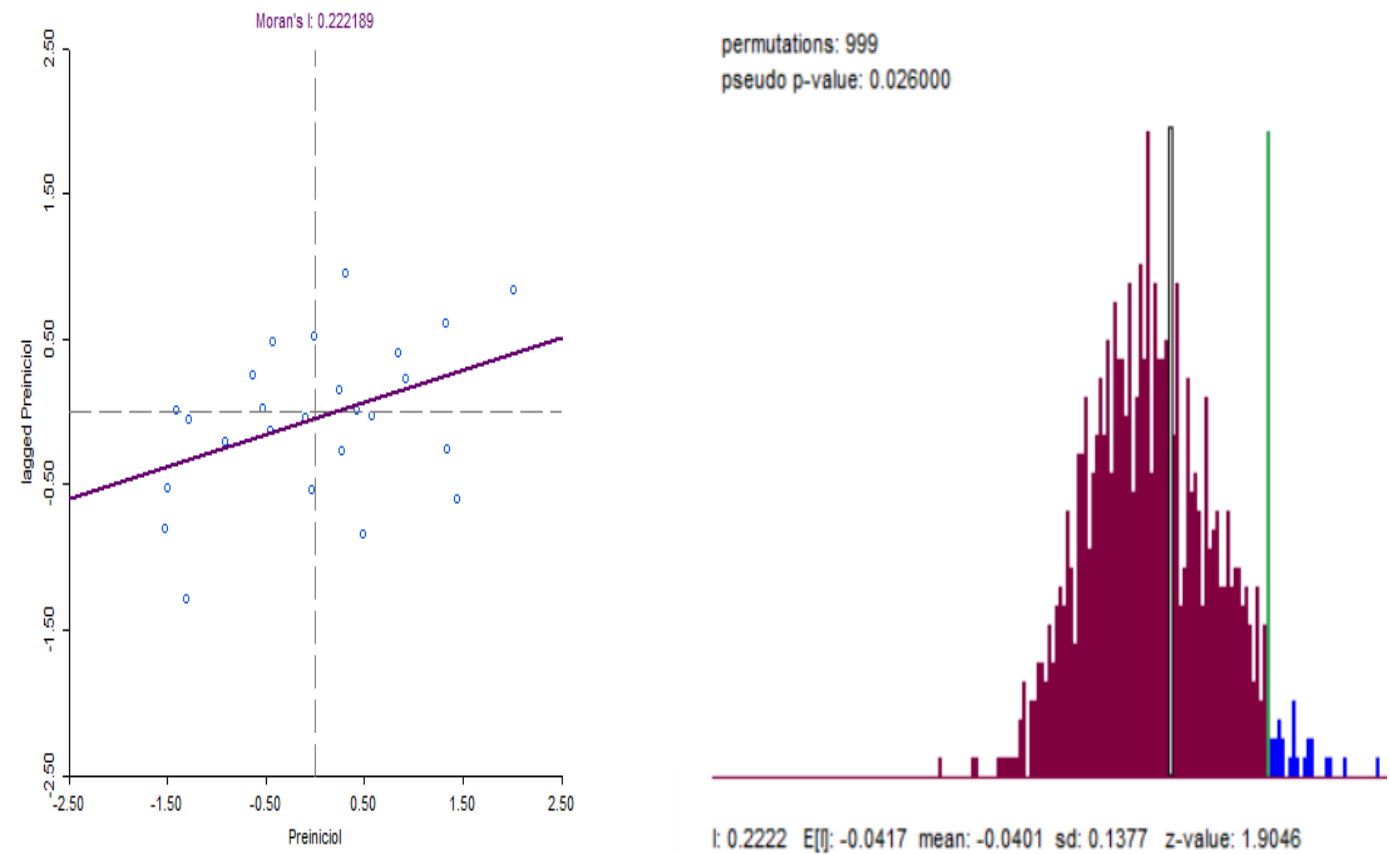

1: 0.2222 E[n: -0.0417 mean: -0.0401 sd: 0.1377 z-value: 1.9046

Figura 9. Diagrama de dispersión del Índice de Moran del nivel de logro de aprendizaje previo al inicio en lectura obtenido por los estudiantes de segundo de secundaria en el Perú - 2018. 

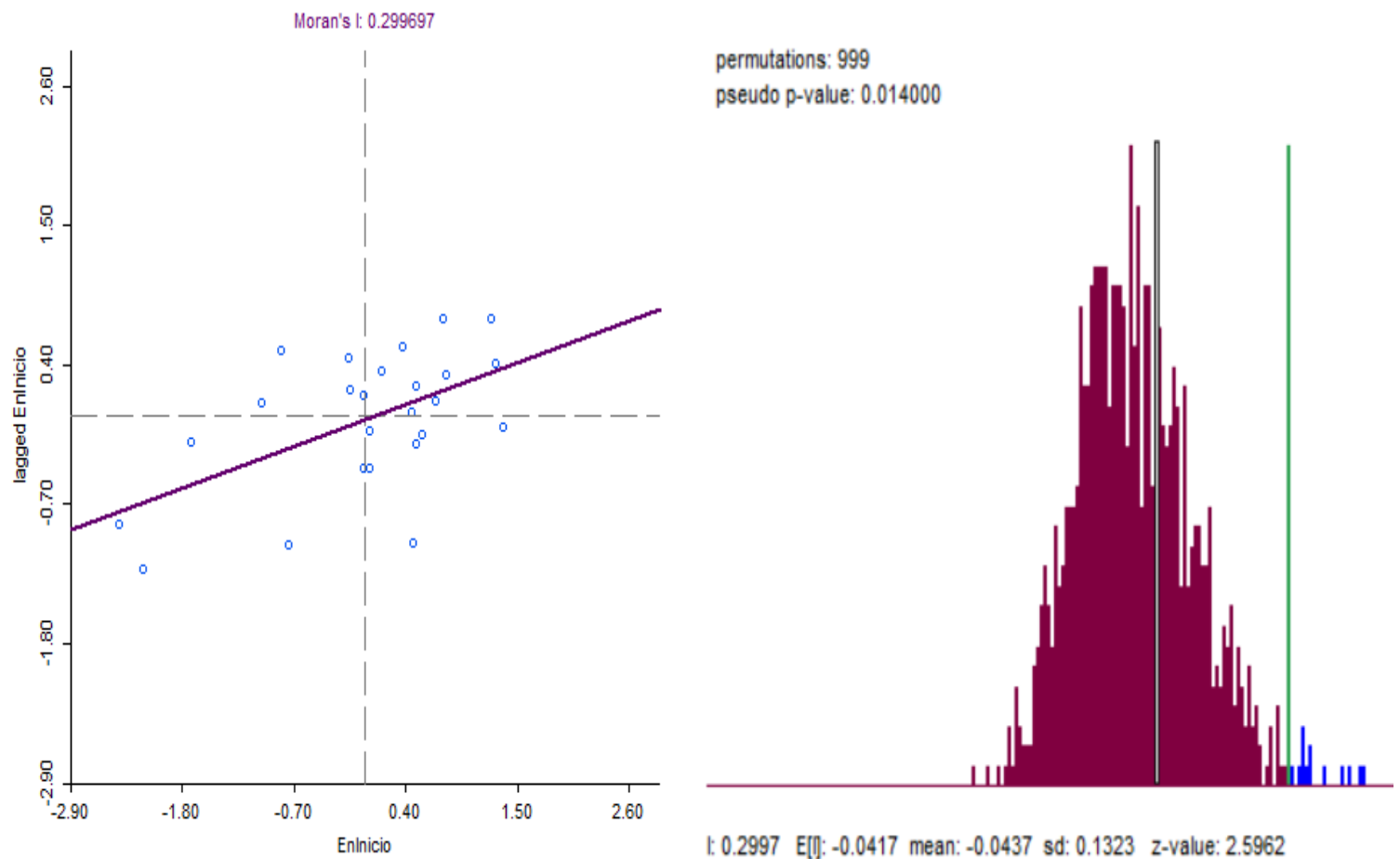

1: 0.2997 E[n]; -0.0417 mean: -0.0437 sd: 0.1323 z-value: 2.5962

Figura 10. Diagrama de dispersión del Índice de Moran del nivel de logro de aprendizaje en inicio en lectura obtenido por los estudiantes de segundo de secundaria en el Perú - 2018.

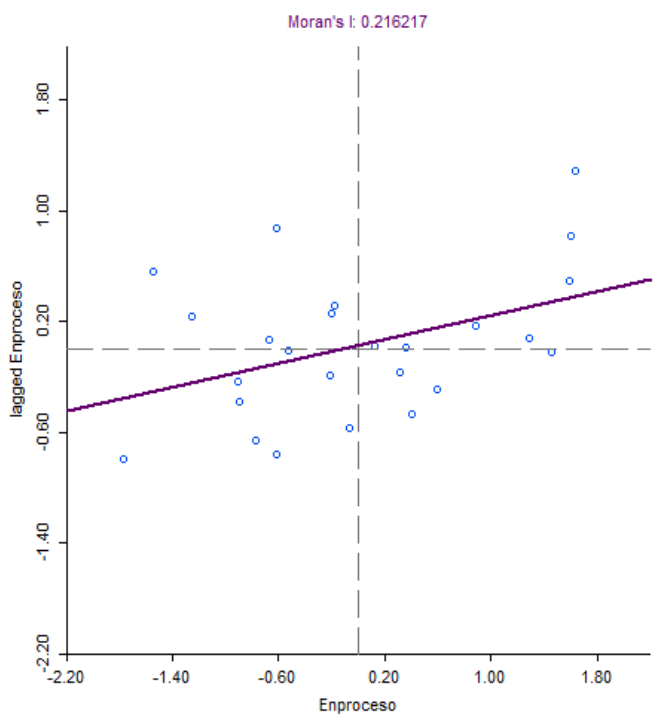

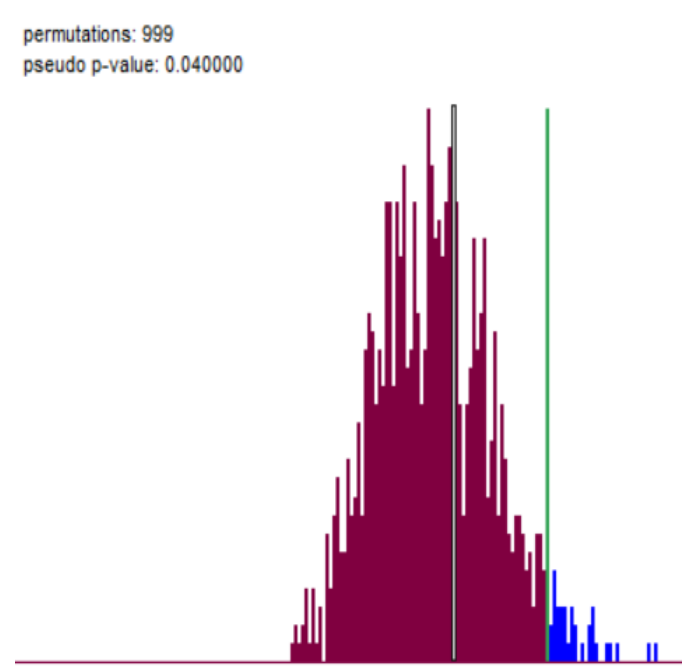

I: 0.2162 E[n: -0.0417 mean: -0.0396 sd: 0.1360 z-value: 1.8803

Figura 11. Diagrama de dispersión del Índice de Moran del nivel de logro de aprendizaje en proceso en lectura obtenido por los estudiantes de segundo de secundaria en el Perú - 2018. 

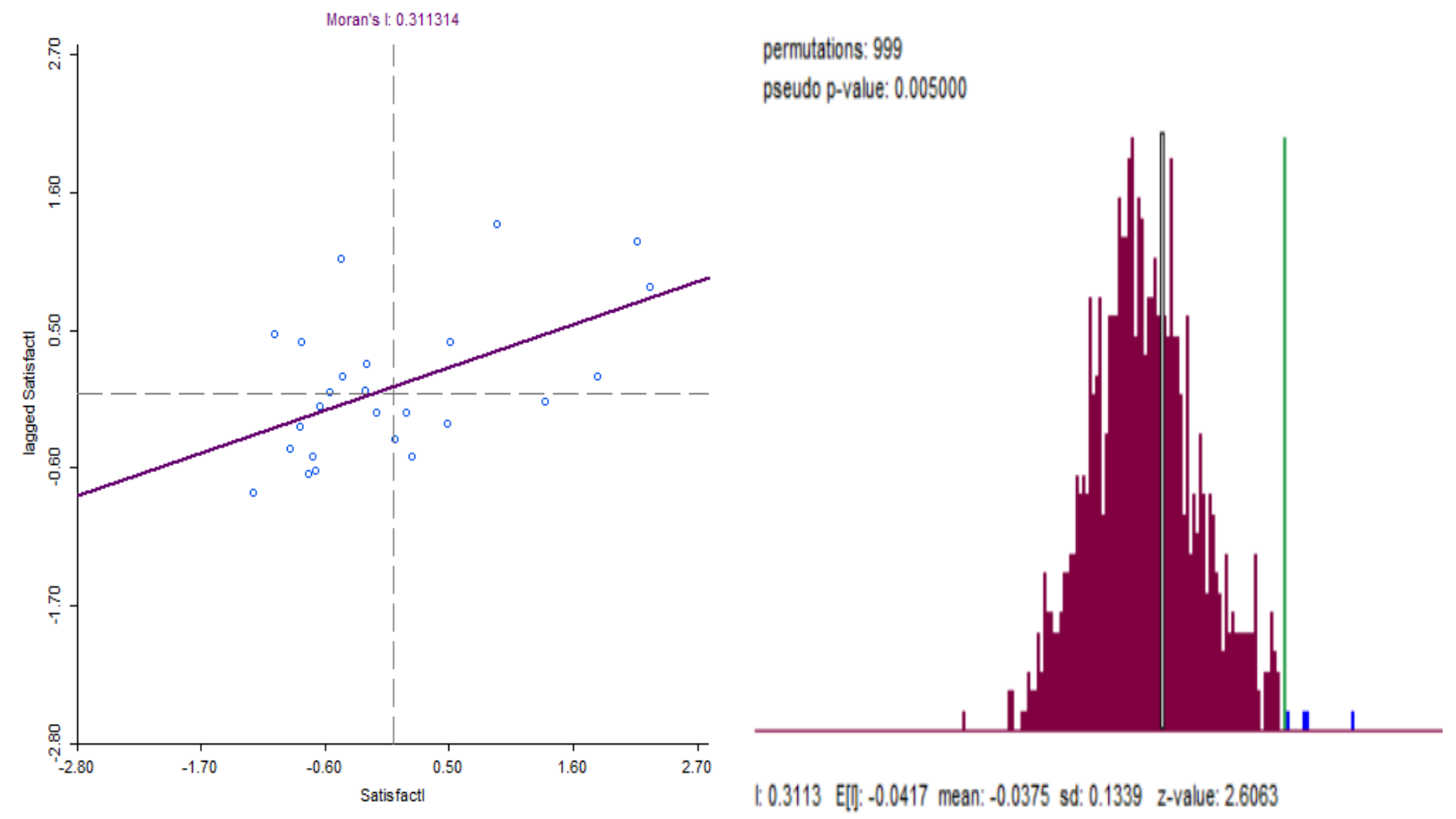

Figura 12. Diagrama de dispersión del Índice de Moran del nivel de logro de aprendizaje satisfactorio en lectura obtenido por los estudiantes de segundo de secundaria en el Perú - 2018

El índice global de autocorrelación espacial de Moran, para los cuatro niveles de aprendizaje en lectura resultaron ser mayores a cero, $0.22,0.30,0.22$ y 0.31 , respectivamente, con p $<0.05$, lo cual demostró que existe autocorrelación espacial con los cuatro niveles de aprendizaje en lectura obtenidos por los dicentes del segundo grado de secundaria en la ECE en las regiones del Perú (ver figuras 9,10,11, y 12). 


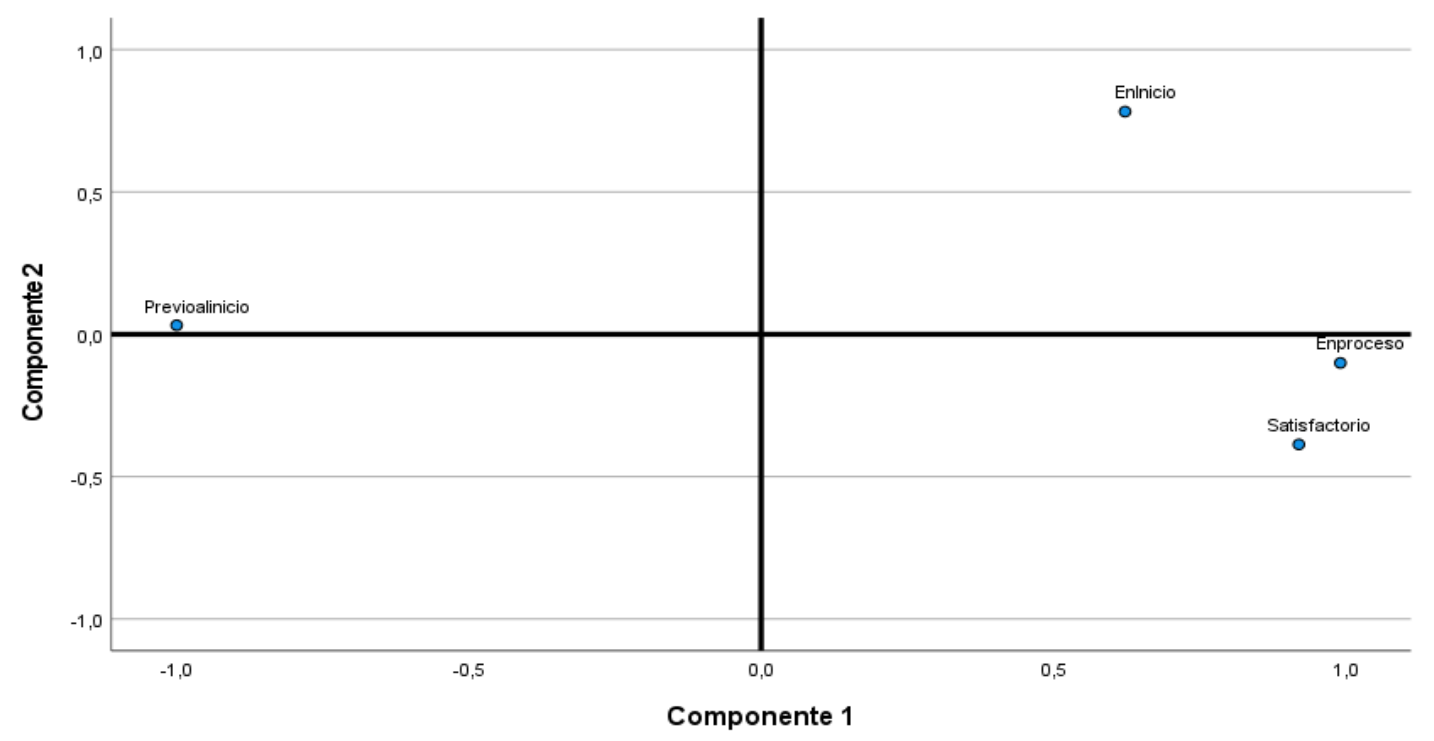

Figura 13. Gráfico de componentes principales considerando el nivel de aprendizaje en matemática en estudiantes de segundo de secundaria en el Perú - 2018

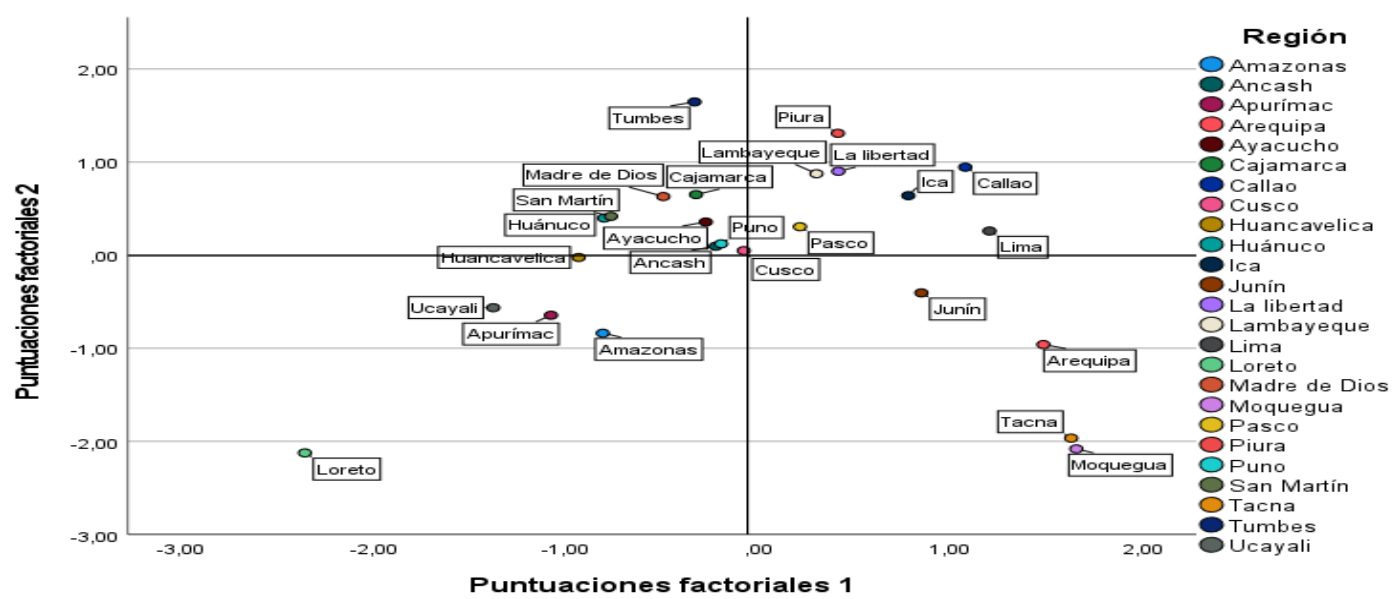

Figura 14. Gráfico de dispersión sobre las puntuaciones factoriales del Análisis de Componentes Principales, del nivel de aprendizaje en matemática en estudiantes de segundo de secundaria en el Perú 2018 


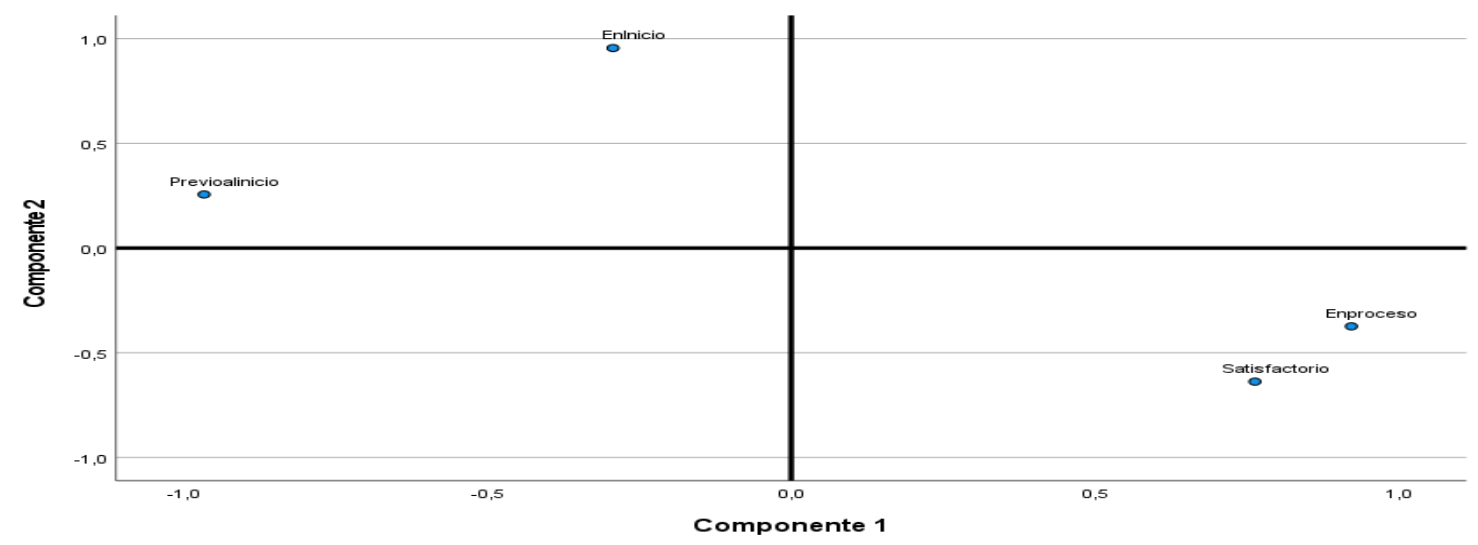

Figura 15. Gráfico de componentes principales rotados del nivel de aprendizaje en lectura en estudiantes de segundo de secundaria en el Perú - 2018

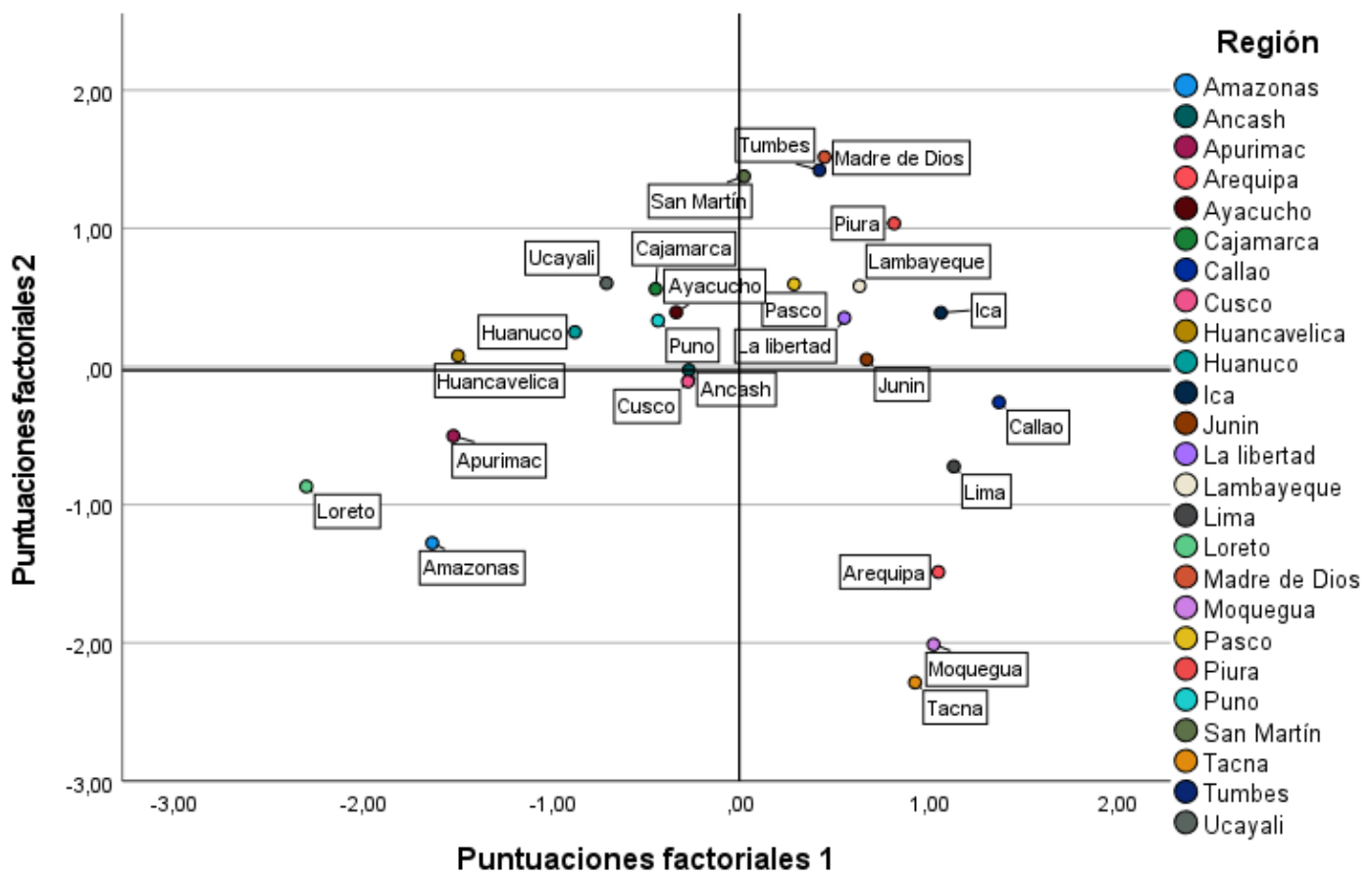

Figura 16. Gráfico de dispersión sobre las puntuaciones factoriales rotadas del Análisis de Componentes Principales, considerando el nivel de aprendizaje en lectura en estudiantes de segundo de secundaria en el Perú - 2018 


\section{Discusión}

Los niveles de logro de aprendizaje en proceso y satisfactorio en matemática obtenidos por los discentes en la ECE durante el año 2018, el mayor porcentaje, corresponden a las regiones de: Lima, Junín, Arequipa, Moquegua, Tacna y Callao. El año 2015, para el nivel de aprendizaje en proceso en matemática, a las regiones consideradas en el año 2018, se excluye la región Junín, mientras que en el nivel de aprendizaje satisfactorio se excluyen las regiones de Callao y Junín. El año 2016, para los niveles de aprendizaje en proceso y satisfactorio en matemática, a las regiones consideradas en el año 2018, se excluye la región Callao. (MINEDU, 2015, 2016). Evidenciándose, que en el año 2018 son más regiones que presentan este comportamiento en los estudiantes.

Los niveles de logro de aprendizaje en proceso y satisfactorio en lectura obtenidos por los discentes en la ECE durante el año 2018, el mayor porcentaje, corresponden a las regiones de: Lima, Ica, Arequipa, Moquegua, Tacna y Callao. El año 2015, para el nivel de aprendizaje en proceso y satisfactorio en lectura, a las regiones consideradas en el año 2018, se excluye la región de Ica. Mientras que, el año 2016, para el nivel de aprendizaje en proceso en lectura, a las regiones consideradas en el año 2018, se excluye la región del Callao, mientras que, en el nivel de aprendizaje satisfactorio en lectura, se excluyen las regiones de Callao e Ica. (MINEDU, 2015, 2016). Evidenciándose, que en el año 2018 son más regiones que presentan este comportamiento en los estudiantes.

Asimismo, el nivel de logro de aprendizaje previo al inicio en matemática obtenidos por los discentes en la ECE durante el año 2018, el mayor porcentaje, corresponden a las regiones de: Loreto, Amazonas, Ucayali, Huánuco, Huancavelica y Apurímac. Resultados similares se encontraron en el año 2016 incluyendo las regiones de Cajamarca, Puno y San Martín; en el año 2015 también se descubrieron los mismos resultados incluyendo las regiones de Ayacucho, Puno y San Martín (MINEDU, 2015, 2016).

Por otro lado, el nivel de logro de aprendizaje en inicio en matemática obtenidos por los discentes en la ECE durante el año 2018, el mayor porcentaje, pertenecen a las regiones: Tumbes, Piura, La Libertad, Lima, Ica y Callao. Para el año 2016, a las regiones consideradas en el año 2018, se añaden la región de Lambayeque. Para el año 2015, a las regiones consideradas en el año 2018, se agregan las regiones de Arequipa, Lambayeque y Moquegua (MINEDU, 2015, 2016). 
Evidenciándose, que en el año 2018 son menos las regiones que presentan este comportamiento en los estudiantes.

En cambio, el nivel de logro de aprendizaje previo al inicio en lectura, durante el año 2018, el mayor porcentaje, corresponde a las regiones: Loreto, Amazonas, Ucayali, Huánuco, Huancavelica y Apurímac. Para el año 2016, a las regiones consideradas en el año 2018, se agregan las regiones de Puno, Cajamarca y Cusco. Mientras que, para el año 2015, a las regiones consideradas en el año 2018, se agregan las regiones de Puno, Ayacucho, Cajamarca y Cusco (MINEDU, 2015, 2016). Evidenciándose, que en el año 2018 son menos las regiones que presentan este comportamiento en los estudiantes.

Por el contrario, el nivel de logro de aprendizaje en inicio en lectura durante el año 2018, el mayor porcentaje, corresponde a las regiones: Tumbes, Piura, Cajamarca, San Martín, Ucayali y Madre de Dios. Para el año 2016, a las regiones consideradas en el año 2018, se agregan las regiones de Puno, Pasco, Lima, Lambayeque, Ica, Ayacucho, Huancavelica y Huánuco. Mientras que, para el año 2015, a las regiones consideradas el año 2018, se agregan las regiones de Ayacucho, Ica, Junín, La Libertad, Lambayeque, Lima y Pasco (MINEDU, 2015, 2016). Evidenciándose, que en el año 2018 son menos las regiones que presentan este comportamiento en los estudiantes.

Entre los resultados más significativos de esta investigación mencionamos que los estudiantes con nivel de aprendizaje previo al inicio en matemática y lectura son aquellos que provienen de las regiones más pobres y extremadamente pobres del Perú en el año 2018 (INEI, 2020), las cuales fueron: Loreto, Amazonas, Huánuco, Huancavelica, Apurímac y Ucayali. Por otro lado, los estudiantes con nivel de aprendizaje en inicio en matemática y lectura son aquellos estudiantes que provienen de regiones más pobres el año 2018. Urge la necesidad de que el gobierno invierta más en educación en las regiones más pobres de nuestro país y en más programas a nivel nacional sobre alimentación escolar, no solo a nivel inicial y primaria sino también a nivel secundaria, el cual debería incluir la alimentación para el turno mañana y tarde.

Es necesario señalar que la presente investigación tuvo algunas limitaciones, no fueron consideradas las áreas de Ciencias Sociales (Historia, Geografía y Economía) y Ciencia Tecnología en los estudiantes de segundo de secundaria de los resultados de la ECE. Asimismo, no fueron considerados los resultados de la ECE de los estudiantes del cuarto grado de primaria y cuarto grado de primaria EIB (Educación Intercultural Bilingüe). 


\section{Conclusión}

El análisis de componentes principales evidenció que los niveles de logro de aprendizaje previo al inicio (-0.966), en proceso (0.922) y satisfactorio (0.763) en lectura, están más asociados al primer componente principal, mientras que el nivel de logro de aprendizaje en inicio (0.955) en lectura está más asociado al segundo componente principal. Por otro lado, los niveles de aprendizaje previo al inicio (-0.999), en proceso (0.991) y satisfactorio (0.920) en matemática están más asociados al primer componente principal, mientras que el nivel de logro en inicio (0.782) en matemática están más asociados al segundo componente principal.

De acuerdo a esto se clasificó a las regiones que tuvieron más relevancia en su nivel de logro de aprendizaje previo al inicio en matemática y lectura, y estas fueron: Loreto (-2.36, -2.30), Amazonas (-0.81, -1.64), Ucayali (-1.38,-0.71), Huánuco (-0.80,-0.88), Huancavelica (-0.93,-1.50) y Apurímac (-1.08,-1.52). El valor del índice de Moran para el nivel de logro de aprendizaje previo al inicio en matemática fue de 0.32 y para lectura fue de 0.22. Se puede concluir que, existe autocorrelación espacial para los niveles de aprendizaje previo al inicio en matemática y lectura, obtenidos por los estudiantes del segundo grado de secundaria en la ECE, el año 2018. (p< 0.05).

Mientras que, las regiones que tuvieron más importancia en su nivel de aprendizaje en inicio en matemática fueron: Tumbes (1.65), Piura (1.31), La Libertad (0.90), Ica (0.64) y Callao (0.95); por el contrario, las regiones que tuvieron mayor relevancia en su nivel de logro de aprendizaje en inicio en lectura fueron: Tumbes (1.42), Piura (1.04), Cajamarca (0.56), San Martín (1.38), Ucayali (0.60) y Madre de Dios (1.52). El valor del índice de Moran para el nivel de logro de aprendizaje en inicio en matemática fue de 0.34, y en lectura fue de 0.30. Se puede concluir que, existe autocorrelación espacial para el nivel de aprendizaje en inicio en matemática y lectura obtenidos por los estudiantes del segundo grado de secundaria en la ECE, el año 2018. (p<0.05).

Sin embargo, las regiones que tuvieron más importancia en sus niveles de aprendizaje en proceso y satisfactorio en matemática y lectura fueron: Lima $(1.20,1.13)$, Ica $(0.78,1.06)$, Arequipa $(1.48,1.05)$, Moquegua $(1.65,1.02)$, Tacna $(1.63,0.92)$, Junín $(0.85,0.67)$ y Callao ( 1.08, 1.37). El valor del índice de Moran para los niveles de logro de aprendizaje en proceso y satisfactorio en matemática fueron 0.31 y 0.36 , respectivamente; mientras que para los niveles de logro de aprendizaje en proceso y satisfactorio en lectura fueron 0.22 y 0.31 , respectivamente. Por lo tanto, se puede concluir que, existe autocorrelación espacial para los niveles de aprendizaje en 
proceso y satisfactorio en matemática y lectura obtenidos por los estudiantes del segundo grado de secundaria en la ECE, el año 2018. (p<0.05).

\section{Referencias}

Acevedo, I. \& Velásquez, E. (2008). Algunos conceptos de la econometría espacial y el análisis exploratorio de datos espaciales. Ecos de Economía, 12 (27), 2-25. https://dialnet.unirioja.es/servlet/articulo?codigo $=4024478$.

Anselin, L. (1995). Local Indicators of Spatial Association-LISA. Geographical Analysis, 27 (2), 93-115. https://doi.org/10.1111/j.1538-4632.1995.tb00338.x

Buzai, G. \& Baxendale, C. (2009). Análisis Exploratorio de Datos Espaciales. Revista digital del Grupo de Estudios sobre Geografía y Análisis Espacial con Sistemas de Información Geográfica (GESIG), Sección Software y Metodología, (1), 1-11. https://ri.unlu.edu.ar/xmlui/bitstream/handle/rediunlu/702/Buzai_An\%C3\%A1lisis\%20Ex ploratorio $\% 20 \mathrm{de} \% 20$ Datos $\% 20$ Espaciales.pdf? sequence $=1 \&$ isAllowed $=\mathrm{y}$

Celemín, J. P. (2009). Autocorrelación especial e indicadores locales de asociación especial, importancia, estructura y aplicación. Revista Universitaria de Geografía, 18 (1), 11-31. https://www.redalyc.org/pdf/3832/383239099001.pdf

Cervera, L. E., Lizárraga, G. M. \& Sánchez, C. P. (2008). Estudio georreferencial de la Evaluación Nacional de Logro Académico en Centros Escolares (ENLACE) en el Municipio de Juárez, Chihuahua: análisis espacial. Revista electrónica de investigación educativa, 10 (1), 1-23. http://www.scielo.org.mx/scielo.php?script=sci_arttext\&pid=S160740412008000100005.

Chasco, C. (2003). Métodos gráficos del análisis exploratorio de datos espaciales. Anales de economía aplicada. Departamento de Economía Aplicada, Universidad Autónoma de Madrid. Madrid. https://www.asepelt.org/ficheros/File/Anales/2003\%20\%20Almeria/asepeltPDF/93.PDF

Chasco, C. (2006). Análisis estadístico de datos geográficos en geomarketing: el programa GeoDa. Distribución y Consumo, 2, 34-45.

Chue, J. (2016). Análisis exploratorio espacial del ingreso de los egresados universitarios del Perú. $\begin{array}{llll}\text { Anales } & \text { Científicos, } & 77 & \text { 329-337. }\end{array}$ http://revistas.lamolina.edu.pe/index.php/acu/article/view/810/pdf_4

De Corso, G. B., Pinilla, M. \& Gallego, J. (2017). Métodos gráficos de análisis exploratorio de datos espaciales con variables espacialmente distribuidas. Cuadernos latinoamericanos de Administración, 13 (25), 92-104. https://doi.org/10.18270/cuaderlam.v13i25.2417 
García, J. (2011). Análisis exploratorio de datos espaciales de la segregación urbana en ciudad Juárez. St. Louis: Federal Reserve Bank of St Louis. https://search.proquest.com/workingpapers/análisis-exploratorio-de-datos-espaciales-la/docview/1698181312/se2 ?accountid $=12268$

Getis, A. \& Ord, J. (1992). The analysis of spatial association by use of distance statistics. Geographical Analysis, 24 (3), 189-206. doi: 10.1111/j.1538-4632.1992.tb00261.x

Griffith, D. A. (2009). Spatial autocorrelation. University of Texas at Dallas, Richardson, TX, Elsevier Inc. http://www.elsevierdirect.com/brochures/hugy/SampleContent/SpatialAutocorrelation

Gordziejczuk, M. A., \& Lucero, P. I. (2019). Turismo y calidad de vida: un estudio de autocorrelación espacial aplicado a la ciudad de Mar del Plata, provincia de Buenos Aires, Argentina. Cuadernos de Geografía: Revista Colombiana de Geografía, 28 (1), 23-42. https://doi.org/10.15446/rcdg.v28n1.67275

Hernández-Sampieri, R. \& Mendoza, C. P. (2018). Metodología de la investigación. Las rutas cuantitativa, cualitativa y mixta. México: McGraw Hill.

INEI (2020). Resultados de la pobreza monetario 2019. https://www.inei.gob.pe/media/MenuRecursivo/boletines/presentacion-del-jefe-del-inei.pdf

Johnson, R. A., \& Wichern, D. (2007). W. Applied multivariate statistical analysis. New Jersey: Prentice Hall Inc.

Levy, J. \& Varela, J. (2003). Análisis multivariable para las ciencias sociales. Madrid: Pearson Educación.

MINEDU (2020). Evaluaciones nacionales de logro de aprendizaje 2019. http://umc.minedu.gob.pe/wp-content/uploads/2020/06/Reporte-Nacional-2019.pdf

MINEDU (2019). Reporte técnico de las evaluaciones censales y muestrales de estudiantes 2018. http://umc.minedu.gob.pe/wp-content/uploads/2019/08/RT-Evaluaciones-2018.pdf.

MINEDU (2018). Sistema de consulta de resultados de evaluaciones (SICRECE). https://sistemas15.minedu.gob.pe:8888/evaluacion_censal_publico

MINEDU (2016). Resultados de la Evaluación Censal de Estudiantes 2016 (ECE 2016). http://umc.minedu.gob.pe/wp-content/uploads/2017/04/ECE-2016-presentaci\%C3\%B3nde-resultados-web.pdf

MINEDU (2015). Resultados de la Evaluación Censal de Estudiantes 2015. http://umc.minedu.gob.pe/wpcontent/uploads/2016/03/Resultados-ECE-2015.pdf

Oyana, T., \& Margai, F. (2015). Spatial analysis. Statistics, visualization, and computatonal methods. New York: CRC Press.

Perez, C. (2014). Análisis multivariante de datos. Madrid: Ibergarceta Publicaciones. 
Rodríguez, F. de J., Pompa-García, M., Hernández-Díaz , C. \& Juárez-Reyes, A. (2010). Patrón de distribución espacial de la pérdida, degradación y recuperación vegetal en Durango, México. Avances en investigación agropecuaria, 14 (1), 53-65. Recuperado de: http://ww.ucol.mx/revaia/anteriores/PDF\%20DE\%20REVISTA/2010/enero/4.pdf

Rodríguez, R. (2017). Software de análisis geoestadístico GEODA. https://risharkygis.wordpress.com/2017/03/23/software-de-analisis-geoestadistico-geoda/.

Serrano, R. M. \& Valcárcel, E. V. (2002). Econometría espacial: Nuevas técnicas para el análisis regional. una aplicación a las regiones europeas. Investigaciones Regionales, (1), 83-106. https://search.proquest.com/scholarly-journals/econometría-espacial-nuevas-técnicaspara-el/docview/1459697470/se-2?accountid=12268

Uriel E. \& Aldás, J. (2005). Análisis multivariante aplicado. Aplicaciones al marketing, Investigación de mercados, Economía, Dirección de empresas y turismo. Madrid: Thomson editores.

Vilalta, C. J. (2005). Cómo enseñar autocorrelación espacial, Economía, Sociedad y Territorio, México, 5 (18), 323-333. https://doi.org/10.22136/est002005307 